\title{
Organic Thin Film Transistors with Gate Dielectrics of Plasma Polymerized Styrene and Vinyl Acetate Thin Films
}

\author{
Jae-Sung Lim \\ HANA Micron Inc., Asan 336-864, Korea \\ Paik-Kyun Shin \\ Department of Electrical Engineering, Inha University, Incheon 402-751 Korea \\ Boong-Joo Lee \\ Department of Electronic Engineering, Namseoul University, Cheonan 330-707, Korea
}

Received March 9, 2015; Accepted April 9, 2015

\begin{abstract}
Organic polymer dielectric thin films of styrene and vinyl acetate were prepared by the plasma polymerization deposition technique and applied for the fabrication of an organic thin film transistor device. The structural properties of the plasma polymerized thin films were characterized by Fourier-transform infrared spectroscopy, X-ray diffraction, atomic force microscopy, and contact angle measurement. Investigation of the electrical properties of the plasma polymerized thin films was carried out by capacitance-voltage and current-voltage measurements. The organic thin film transistor device with gate dielectric of the plasma polymerized thin film revealed a low operation voltage of -10 $\mathrm{V}$ and a low threshold voltage of $-3 \mathrm{~V}$. It was confirmed that plasma polymerized thin films of styrene and vinyl acetate could be applied to functional organic thin film transistor devices as the gate dielectric.
\end{abstract}

Keywords: Plasma polymerization, Organic thin film transistor, Gate dielectric, Plasma polymerized styrene (ppS), Plasma polymerized vinyl acetate (ppVA)

\section{INTRODUCTION}

Organic thin film transistors (OTFTs) are representative organic electronic devices with flexible characteristic and low-cost process compatibility, and are often employed as sensors, display backplane, and rectifiers for radio frequency identification [1]. Recent research on OTFTs have been devoted to achieving low operation voltage to allow reduction of power consumption.

${ }^{\dagger}$ Author to whom all correspondence should be addressed: E-mail: bjlee@nsu.ac.kr

Copyright $@ 2015$ KIEEME. All rights reserved.

This is an open-access article distributed under the terms of the Creative Commons Attribution Non-Commercia

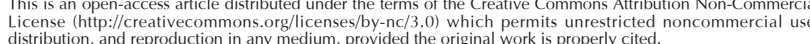

High operation voltage is mainly caused by inferior permittivity/capacitance properties conferred by relatively thick polymer dielectrics, which could be solved by high- $k$ and/or thin polymer dielectric. Moreover, OTFTs can be fabricated using a lowtemperature-process to allow preparation of electronic circuits and systems on flexible large-area substrates. Several techniques have also been developed, adapted, and extended to the set of fabrication techniques for polymer dielectrics. Most of the related studies have focused on developing efficient fabrication processes for achievement of high performance OTFT devices. Polymer dielectrics can be deposited by solution processes such as spin-coating, dip coating, micro-contact printing, and inkjet printing [1]. However, these wet processes can possibly cause overall degradation of the device performance due to damage to the organic layer by the solvents involved in the lift-off process, 

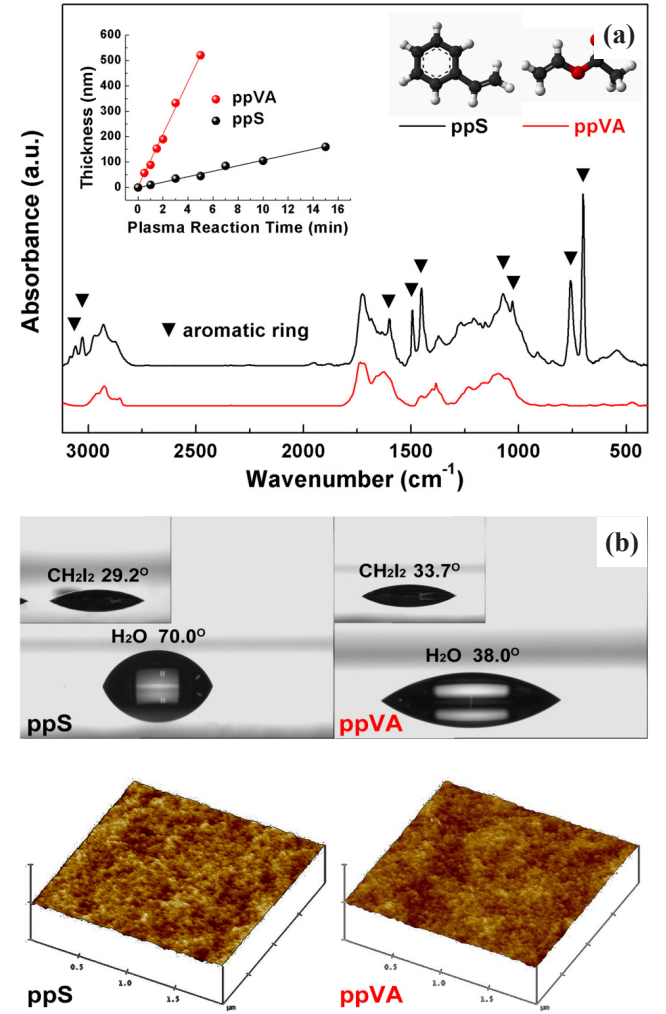

Fig. 1. (a) FT-IR spectra of the plasma polymer layers. Inset: thickness of the plasma polymers as a function of the plasma reaction time, and chemical structure of styrene and vinyl acetate monomers and (b) atomic force microscopy images $(2 \mu \mathrm{m} \times 2 \mu \mathrm{m})$ and optical microscopy images of distilled water and diiodomethane drops on the surface of $100 \mathrm{~nm}$-thick plasma polymer layers.

or attack of the other layer during thermal treatment. Use of a dry fabrication process for polymer dielectrics might be a solution to avoid such problems.

The plasma polymerization technique is another alternative for the preparation of polymer dielectrics. Plasma polymerized polymers show excellent coating adhesion to any substrate, chemical/mechanical/thermal stability, high barrier effect, are pin-hole free and have flat surface structure formability. Most plasma polymers, which are distinguished from conventional polymers, consist of a structure that lies somewhere between the polymeric and inorganic materials, and is in the form of a highly cross-linked and highly branched three-dimensional network [2].

In the present study, a plasma polymerization method was proposed for application to a completely dry process for OTFT fabrication, which offers a one-step and a compatible process with the conventional process technologies. The OTFT devices had inverted staggered structure of Au/pentacene/plasma polymers/ITO. The effects of plasma polymerized polymer dielectric layers were studied for realization of low operation voltage of the OTFTs. Hydrophobic styrene and hydrophilic vinyl acetate [see Fig. 1(a) inset] monomers were used for the plasma polymerization of polymer dielectric layers.

\section{EXPERIMENTAL DETAILS}

ITO $\left(150 \mathrm{~nm}, 12 \Omega / \mathrm{cm}^{2}\right)$ coated glasses were used as substrates. The substrates were ultrasonically cleaned in acetone, ethanol, and distilled water, allowing removal of most of the organic contaminants on the ITO surface. Radio frequency (r.f.) capacitively-coupled plasma (CCP) was used for surface treatment of the ITO, with a system pressure of 0.1 Torr, Ar gas flow rate of $10 \mathrm{sccm}$, and main power of $100 \mathrm{~W}$, carried out at room temperature. Without breaking the vacuum after plasma surface treatment, the plasma polymer layers were then deposited. Plasma polymerization of the styrene and vinyl acetate monomers was again done at room temperature using the CCP equipment. A detailed survey of the plasma polymerization processes can be found in various reviews and books, such as in references [2-5]. The plasma polymers were deposited under the following experimental conditions [see Fig. 1(a) inset]: main power of $200 \mathrm{~W} /$ bias power of $10 \mathrm{~W}$, system pressure of $0.5 \mathrm{Torr}$, Ar gas flow rate of $30 \mathrm{sccm}$, and deposition time of 1-10 min through a shadow mask. The thickness was measured by evaluating micrographs of cross-sections of the plasma polymers using field emissionscanning microscopy (FE-SEM; Hitachi, S-430), and was found to be approximately $100 \mathrm{~nm}$. A $70 \mathrm{~nm}$-thick pentacene layer, as the active channel layer, and $100 \mathrm{~nm}$-thick Au as the source/ drain electrodes were then deposited using the thermal evaporation technique. The Au source/drain electrodes were patterned on the pentacene layer through a shadow mask.

Film composition, surface morphology, and electrical properties of the plasma polymers were investigated using Fourier transform infrared spectroscopy (FT-IR; Bruker Optics, Vertex $80 \mathrm{v}$ ) in a vacuum, contact angle measurement (Erma inc.), Xray diffraction (XRD; Rigaku, M/Max 2,200 V/PC), atomic force microscopy (AFM; Digital instrument, Nanoscope Mutimode IV a) and a C-V analyzer (Keithley 590). To investigate the performance of the OTFTs, the current-voltage (I-V) characteristics were measured according to the applied voltage change using a source measurement unit (Keithley 2400) combined with another source measurement unit (Keithley 236).

\section{RESULTS AND DISCUCSSION}

Figure 1(a) shows the FT-IR spectra of the plasma polymerized styrene (ppS) and plasma polymerized vinyl acetate (ppVA) layers. The strongest absorptions of the ppS layer occurred for aromatic compounds in the region of $900 \sim 650 \mathrm{~cm}^{-1}$, due to $\mathrm{C}-\mathrm{H}$ vibrations out of the plane of the aromatic ring. Most mono-nuclear and poly-nuclear aromatic compounds have peaks in the region of 3,080 3,010 $\mathrm{cm}^{-1}$, due to the stretching vibrations of the ring $\mathrm{C}-\mathrm{H}$ bonds. Ring carbon-carbon stretching vibrations occur in the region of 1,625 1,430 $\mathrm{cm}^{-1}$. The formation of benzene rings in the ppS was likely due to non-destruction of the aromatic structure as a result of plasma bombardment. In the ppS and ppVA, the characteristic peaks for the conventional polymers, i.e. polystyrene and polyvinylacetate, showed comparatively weak and broader (but not absent) absorbance, suggesting significant amounts of fragmentation and rearrangement during the plasma deposition process. This may result in the formation of pinholefree plasma polymer layers with highly cross-linked structure. Similar results were revealed by the FT-IR spectra of the plasma polymers, which identified chemical similarities and dissimilarities of the two materials as a result of a strongly system-dependent process. Figure 1(b) shows the contact angle measurements and AFM 3D-images of the plasma polymers. The surface energy of the ppS and ppVA layers was calculated from the contact angle measurements using distilled water $\left(70.0^{\circ} \pm 2 \%\right.$ and $38.0^{\circ}$ $\pm 2 \%)$ and diiodomethane $\left(29.2^{\circ} \pm 2 \%\right.$ and $\left.33.7^{\circ} \pm 2 \%\right)$ as the probe liquids. The geometric mean equation was used to calculate the surface energy [5]: 


$$
(1+\cos \theta) \gamma_{p l}=2\left(\gamma_{s}^{d} \gamma_{p l}^{d}\right)^{1 / 2}+2\left(\gamma_{s}^{p} \gamma_{p l}^{p}\right)^{1 / 2}
$$

The surface energy of the ppS and ppVA layers was found to be approximately 45 and $64 \mathrm{~mJ} / \mathrm{m}^{2}$, respectively. The lower surface energy of the ppS could be thought to be attributable to the higher density of the aromatic ring [6,7]. The AFM 3D-images of the $100 \mathrm{~nm}$-thick layers of both ppS and ppVA grown on ITO are shown in Fig. 1(b). The root mean square (RMS) roughness of the ppS and ppVA layers was found to be approximately 0.296 and $0.265 \mathrm{~nm}$, respectively. It could be seen that the plasma polymers had very flat surface structures with pin-hole free formability.

The capacitance of the gate dielectric, i.e. the thickness and dielectric constant, determine the operating voltage of the OTFTs [8-10]. The dielectric constants, $k$, of the plasma polymers were obtained by conventional methods, with placement of the ppS (3.66) and ppVA (5.84) dielectrics in an ITO-plasma polymer-Au (metal-insulator-metal; MIM) sandwich structure [11]. To investigate the feasibility of application of the plasma polymers as a gate dielectric, we checked whether the leakage current density was sufficiently low by testing the corresponding MIM devices.

Figure 2 shows the x-ray diffraction (XRD) patterns and AFM image of the $70 \mathrm{~nm}$-thick pentacene grown on the plasma polymer dielectrics. The XRD and AFM analyses were performed in order to investigate the effects of the surface properties of the plasma polymer dielectrics on the surface morphology of the pentacene. The corresponding XRD pattern contained a series of sharp $(00 \mathrm{k})$ peaks, indicating that the pentacene film was highly ordered. The first peak at $2 \theta=5.7^{\circ}$ (the thin-film phase) corresponded to the lattice parameter of $15.6 \AA$. The AFM image of pentacene on the ppS dielectric revealed a dendritic structure with a grain size of approximately $1 \mu \mathrm{m}$. The growth morphology of pentacene on the plasma polymers was similar to those observed on other polymer gate dielectrics [12].

Figure 3 (a) shows the $C$ - $V$ characteristics ( $f=100 \mathrm{kHz}$ ) of the ITO-plasma polymer-pentacene (metal-insulator-metal; MIS) devices prepared with $100 \mathrm{~nm}$-thick plasma polymers in the dark at room temperature. The normalized capacitance $(\mathrm{C} /$ $C_{\text {MAX }}$ ) ranged between 1.00 0.80 and 1.00 0.65 when the applied voltage was varied from -20 to $20 \mathrm{~V}$. The $C-V$ characteristics in the accumulation-depletion region were investigated using the MIS capacitors, and the gate bias was swept back to check the amount of hysteresis origination from the bulk or interface charge trapping of the plasma polymer dielectrics. One of the main problems of OTFT devices is the occurrence of hysteresis effect. The MIS device fabricated with ppVA dielectric revealed a larger hysteresis loop, which disappeared when the MIS device with the ppS dielectric was used. Lee et al. [13] reported a correlation between the bulk/interface states of polymer dielectrics and the $C$ - $V$ and $I$ - $V$ outputs of polymer dielectrics, determining that larger hysteresis was caused by the density of hydroxyl groups contained in the monomer. Moreover, negligible hysteresis was observed in the $C$ - $V$ curves of the MIS device employing the ppS dielectric, which was attributed to a lack of bulk and interface states. This might be caused by the defect-free structure of the ppS dielectric, with high cross-linking and no pinholes [14]. The OTFT with the ppVA dielectric showed inferior performance, presumably because of the density of hydroxyl groups remaining at the bulk and surface of the ppVA. Figure 3(b) shows the current density-electric field (J-E) curve of the plasma polymers on ITO. In particular, the leakage behavior of the plasma polymer dielectrics can be seen in the figure. The ppS appeared to have quite a good dielectric strength of $\sim 5 \mathrm{MV} / \mathrm{cm}$ based on the minimum leakage current density of $\sim 10^{-7} \mathrm{~A} / \mathrm{cm}^{2}$, even though its

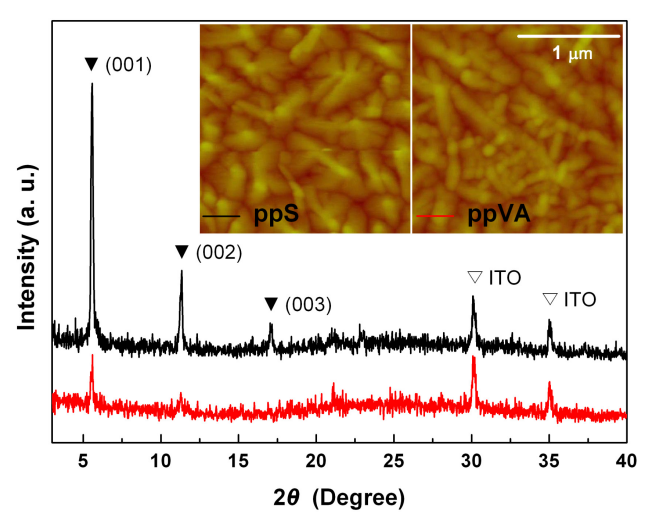

Fig. 2. X-ray diffraction patterns of the pentacene deposited on the plasma polymer layers. Inset: Atomic force microscopy images $(2 \mu \mathrm{m}$ $\times 2 \mu \mathrm{m}$ ) of the pentacene grown on the plasma polymer layers.
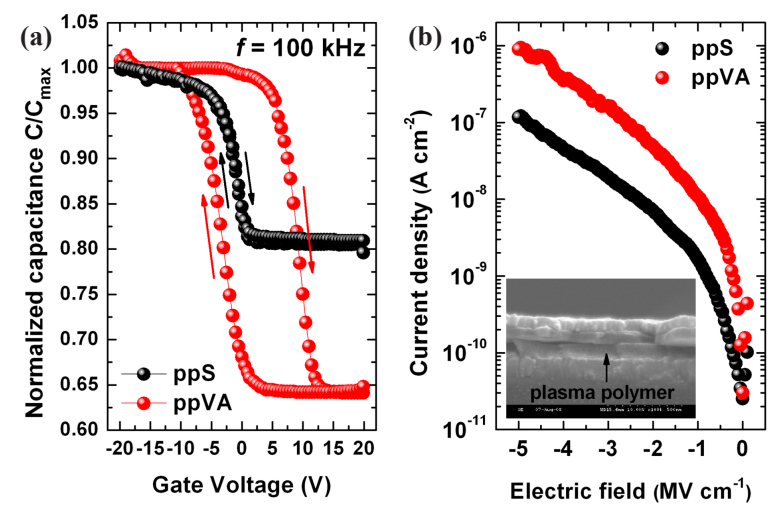

Fig. 3. (a) $C$ - $V$ characteristics of ITO-plasma polymers-pentacene (MIS) capacitors at AC signal frequency $(100 \mathrm{kHz})$. The bias voltages were swept from +20 to $-20 \mathrm{~V}$, and from -20 to $+20 \mathrm{~V}$ and (b) J-E characteristics of the ITO-plasma polymers-Au (MIM) device. Inset: cross sectional image of the MIS device using field emission-scanning electron microscopy.

total thickness was only $100 \mathrm{~nm}$. This may be due to the pinholefree structure of the ppS dielectric, with high cross-linking. Figure 4 shows the $I-V$ characteristics of the OTFT devices with a 100 $\mathrm{nm}$-thick-plasma polymer layer in the dark at room temperature for transfer $I_{\mathrm{D}}-V_{\mathrm{G}}$ curves at the fixed drain voltage of $-10 \mathrm{~V}$. The $I-V$ characteristics were measured for the OTFT device with the channel length of $100 \mu \mathrm{m}$ and the channel width of 2,000 $\mu \mathrm{m}$. From these data, the average field-effect mobility was calculated in the saturation current regime $\left(V_{\mathrm{D}}=-10 \mathrm{~V}\right)$ using the following equation [15]:

$$
I_{\text {Dsat }}=\frac{W}{2 L} C_{i} \mu\left(V_{G}-V_{T H}\right)^{2}
$$

where $W$ and $L$ are the channel width and length, respectively, $C_{\mathrm{i}}$ is the capacitance per unit area of the ppS insulators, $V_{\mathrm{G}}$ and $V_{\mathrm{TH}}$ are the gate and threshold voltage, respectively, and $\mu$ is the field effect mobility. The OTFT device with the ppS dielectric revealed the field effect mobility of $0.08 \mathrm{~cm}^{2} \mathrm{~V}^{1} \mathrm{~s}^{-1}$, the sub-threshold slope of $0.985 \mathrm{~V} /$ decade, the on/off current ratio $\left(I_{\text {on }} / I_{\text {off }}\right)$ of $1 \times 10^{4}$, and the threshold voltage of $-3.0 \mathrm{~V}$. For the OTFT with the 
(a)

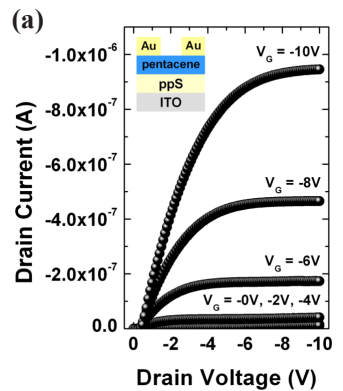

(c)

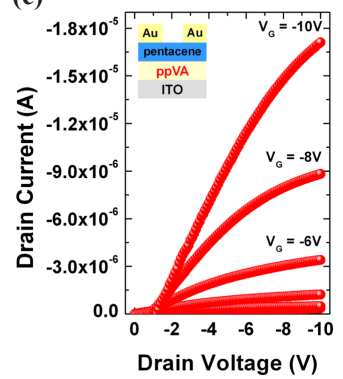

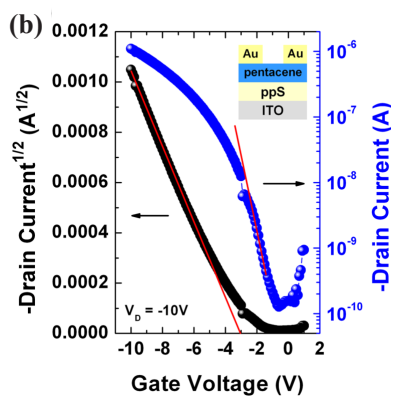

(d)

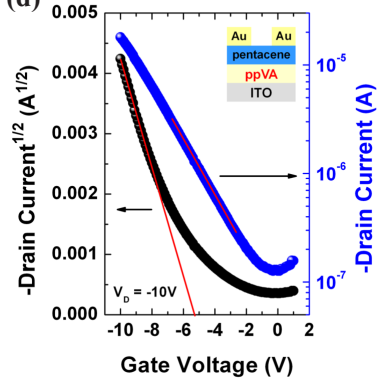

Fig. 4. Output characteristics obtained from the OTFT devices which included (a) ppS dielectrics, (b) ppVA dielectrics. Transfer characteristics of the OTFT devices using, (c) ppS dielectrics, and (d) ppVA dielectrics. Inset: schematic cross section of the OTFT device.

ppVA dielectric, the following parameters were achieved: field effect mobility of $1.54 \mathrm{~cm}^{2} \mathrm{~V}^{-1} \mathrm{~s}^{-1}$, sub-threshold slope of $0.247 \mathrm{~V} /$ decade, on/off current ratio $\left(I_{\text {on }} / I_{\text {off }}\right)$ of $9 \times 103$, and threshold voltage of $-5.5 \mathrm{~V}$. Higher mobility could be achieved for the OFET device with the ppVA due to the relatively higher dielectric constant. It is thought that the gate voltage dependence was a result of the higher concentration of the charge carriers accumulated in the channel, achieved through use of these dielectric $[13,16]$. This combination of favorable properties demonstrated that the OTFT can be operated successfully at voltages up to approximately $10 \mathrm{~V}$. The standard deviation of the device parameters was approximately $\pm 15 \%$.

\section{CONCLUSIONS}

In summary, OTFT with plasma polymer dielectrics having high capacitive coupling could be operated at the low operation voltage of $-10 \mathrm{~V}$. The threshold and operating voltages were the lowest among those reported for polymer gate dielectrics. It was

demonstrated herein that the plasma polymer dielectrics could be used in the fabrication of OTFT devices with favorable electrical properties, allowing significant improvement in the performance of the OTFT device. We believe that the proposed method provides an effective way to improve the electrical properties of plasma polymer dielectrics for OTFT devices, possibly by substituting the polymer dielectric through co-polymerization or double-layered structure.

\section{ACKNOWLEDGMENT}

This study was supported by the fund of Namseoul University.

\section{REFERENCES}

[1] H. Klauk, Organic Electronics, Materials, Manufacturing and Applications (Wiley-VCH Weinheim, 2006) Chapter 6.

[2] H. K. Yasuda, Plasma Polymerization (Academic press, New York, 1985) Chapter 4.

[3] J. S. Lim and P. K. Shin, Appl. Surf. Sci., 253, 3828 (2007).

[4] S. P. Russell and D. H. Weinkauf, Polymer, 42, 2827 (2001).

[5] F. M. Fowkes, Treatise on Adhesion and Adhesives, edited by R. L. Patrick Marcel Dekker (New York, 1967) p. 352.

[6] H. L. Luo, J. Sheng, and Y. Z. Wan, Appl. Surf. Sci., 253, 5203 (2007).

[7] D. Merche, C. Poleunis, P. Bertrand, M. Sferrazza, and F. Reniers, IEEE Trans. Plasma Sci., 37, 951 (2009).

[8] M. Halik, H. Klauk, U. Zschieschang, G. Schmid, C. Dehm, M. Schutz, S. Maisch, F. Effenberger, M. Brunnbauer, and F. Stellacci, Nature, 431, 963 (2004).

[9] H. Klauk, M. Halik, F. Eder, G. Schmid, C. Dehm, D. Rohde, R. Brederlow, S. Briole, S. Maisch, F. Effenberger, and U. Zschieschang, Electron Devices Meeting (IEDM) Tech. Dig., 369 (2004).

[10] C. D. Dimitrakopoulos, S. Purushothaman, J. Kymissis, A. Callegari, and J. M. Shaw, Science, 283, 822 (1999).

[11] N. Arora, MOSFET Models for VLSI Circuit Simulation Theory and Practice (Springer, New York, 1993) Chapter 4.

[12] S. Y. Yang, K. Shin, and C. E. Park, Adv. Funct. Mater., 15, 1806 (2005).

[13] S. Lee, B. Koo, J. Shin, E. Lee, H. Park, and H. Kim, Appl. Phys. Lett., 88, 162109 (2006).

[14] J. S. Lim, P. K. Shin, B. J. Lee, and S. Lee, Org. Electron., 11, 951 (2010).

[15] N. Arora, MOSFET Models for VLSI Circuit Simulation Theory and Practice (Springer, New York, 1993) Chapter 7.

[16] Y. Jang, D. H. Kim, Y. D. Park, J. H. Cho, M. Hwang, and K. Cho, Appl. Phys. Lett., 87, 152105 (2005). 